\title{
The Effect of Amplitude Modulated Contralateral Signals on Distortion Product Otoacoustic Emissions
}

\author{
A. WICHER* \\ Institute of Acoustics, Faculty of Physics, A. Mickiewicz University \\ Umultowska 85, 61-614 Poznań, Poland
}

\begin{abstract}
Contralateral stimulation of the auditory system causes changes in levels of otoacoustic emissions. The main objective of this study was to determine the changes in the level of distortion product otoacoustic emissions for contralaterally presented unmodulated broad-band noise, or amplitude modulated broad-band noise. Two types of modulating signal were used in the investigations (sine or rectangular wave form). The modulation depth was $100 \%$ for both the sinusoidal and the rectangular modulation. The modulation rate was 4 or $100 \mathrm{~Hz}$. The generation and acquisition of the distortion product otoacoustic emissions signal lasted $2 \mathrm{~s}$. The values of the distortion product otoacoustic emissions level changes were defined as the difference between the mean distortion product otoacoustic emissions levels with and without contralateral stimulation. Ten normal-hearing subjects participated in this study. The results showed that reduction in the level of the distortion product otoacoustic emissions (suppression effect) was highest for the low $F_{2}$ frequencies and decreased along with the increase in the $F_{2}$ frequency. The modulation type of the contralateral stimulation did not influence the mean suppression effect significantly. However, the distortion product otoacoustic emissions level reduction reached higher values for the unmodulated contralateral stimulation than for the modulated, and these differences were statistically significant.
\end{abstract}

PACS: $43.64 . J b$

\section{Introduction}

The distortion products otoacoustic emissions (DPOAEs) are characterized by the appearance of non-linear distortions when two primary tones of the frequencies $F_{1}$ and $F_{2}$, and the levels $L_{1}$ and $L_{2}$, are delivered to the ear canal. The highest registered level for these distortions occurs for the frequency $F_{\mathrm{DP}}=2 F_{1}-F_{2}$. These distortions concern nonlinear mechanisms inside the cochlea which are associated with the outer hair cells (OHCs) function. DPOAE measurements enable the objective evaluation of human cochlear functioning. They are applied in clinical examinations in order to diagnose the inner ear. Changes in $\mathrm{OHC}$ functioning are always reflected in changes of the DPOAE level [1, 2].

The nerve fibers in the medial olivocochlear system (MOC) directly innervate the OHCs [3]. This connection refers to the efferent part of the auditory system. Stimulation of the efferent system can influence the OHCs functioning.

Contralateral stimulation (CS) of the auditory system is one of the types of auditory activation and enables an analysis of functioning of the efferent auditory pathways $[4-6]$. When the DPOAEs are measured in the

* e-mail: awaku@amu.edu.pl presence of additional CS, changes in the DPOAE level are observed [7-9]. Contralateral stimulation most often causes a reduction in the DPOAE level (from 0.5 to $2 \mathrm{~dB}$ ) [10-13], however sometimes an increase in the level of DPOAEs is observed [13, 14]. Reduction in the level of otoacoustic emission (OAE) is known as the suppression phenomenon [6]. A change in the value of DPOAE depends on both the type and level of CS. The most effective $\mathrm{CS}$ is wideband noise (WBN). Many authors prove that using wideband contralateral signals produces a significant reduction in the level of the $\operatorname{OAE}[15,16]$. When the CS is a noise band or tonal signal, changes in the level of otoacoustic emissions are lower or do not occur at all $[5,15]$. Increasing the level of CS causes the suppression effect to be increased [17]. Changes in the level of the OAE evoked by CS are often used as an indicator of efferent activity [18]. The opposite effect, i.e. of enhancement, was observed only in a low percentage of cases $[3,5,13,14]$.

There are not many papers concerning the problem of the contralateral signal modulation on the OAE level changes [10, 19-21]. Maison et al. [19] applied transient-evoked otoacoustic emissions (TEOAE) and used a frequency modulated (FM) tone as a CS. Their results showed that TEOAE amplitude was reduced by contralateral FM signals, with significant influences from both the modulation rate and modulation depth. They demonstrated that TEOAE suppression increased for the 
larger frequency deviation values. The results can be compared to the phenomenon of increasing suppression accompanying the increasing bandwidth of the CS [5].

Micheyl et al. [20] applied harmonic complex tones as the $\mathrm{CS}$, with the fundamental component $F_{0}$ varying in octave steps within the range from $50 \mathrm{~Hz}$ to $400 \mathrm{~Hz}$. They found that harmonic complex tones are as effective as a suppressor as broadband noise. Furthermore, the TEOAE suppression effect is strongly dependent on the $F_{0}$ value. The reduction in TEOAE amplitude is largest at $F_{0}$ 's around $100-200 \mathrm{~Hz}$ and decreases at lower and higher $F_{0}$ 's. Overall, Micheyl et al. [20] stated that the amount of contralateral suppression depends on both the number and relative size of the peaks in the stimulus wave form.

In another paper [21], a similar problem of the influence of modulated CS on the TEOAE level was brought up. Otoacoustic emissions were recorded in normal hearing subjects, successively in the absence and presence of contralateral noise that was either steady or modulated sinusoidally, in amplitude at depths from 25 to $100 \%$, and with modulation rates from 50 to $800 \mathrm{~Hz}$ in half-octave steps. The results obtained by Maison et al. [21] showed that the TEOAE suppression effect induced by CS varied depending on the modulation depth and modulation rate of the contralateral amplitude-modulated noise. The largest suppression effect was observed at the $100 \mathrm{~Hz}$ modulation rate and $100 \%$ of modulation depth.

James et al. [10] measured contralateral suppression of DPOAE in humans. They found that the magnitude of suppression increased with contralateral stimulus intensity and the onset latency of suppression was around $43 \mathrm{~ms}$ (31-95 ms). However Harrison et al. [22] stated that the onset latency of suppression was $25 \mathrm{~ms}$. They used broadband noise stimuli as CS, presented at approximately $30 \mathrm{~dB}$ SPL (sound pressure level) and increased or decreased from the baseline in 10 or $20 \mathrm{~dB}$ steps. Additionally, they observed that DPOAE amplitude closely follows contralateral amplitude signals up to modulation rates of approximately $20 \mathrm{~Hz}$.

So far research has not been conducted on the scope of the influence of amplitude modulated contralateral signals on the DPOAE level in a wide range of $F_{2}$ frequencies and for different types of modulating signals. The main objective of the present study was to determine changes in the level of DPOAE for contralaterally presented unmodulated broad-band noise or amplitude modulated broad-band noise. Two types of modulating signal were used in the investigations (sine or rectangular wave form).

\section{Materials and methods}

Ten normal-hearing subjects took part in this study. Their ages ranged from 19 to 26 years old. The tonal audiometry, determined by means of a clinical audiometer (AC 40 Interacoustics), referred to measurements of hearing thresholds at standard frequencies from 125 to
$8000 \mathrm{~Hz}$. Subjects had hearing thresholds at or better than $15 \mathrm{~dB}$ HL (hearing level) in both ears, and they had no history of ear diseases. The middle ear testing, performed by means of a Homoth tympanometer, revealed normal middle ear function for all subjects.

The DPOAE measurements were performed by applying analogous methods to those in the [13] investigations. A Tucker-Davis Technologies (TDT) System 3 was applied in the DPOAE measurements. Two primary tones at frequencies $F_{1}$ and $F_{2}\left(F_{2} / F_{1}=1.22\right)$ and the contralateral signal were generated by a 24 -bit digital real-time signal processor RP2.1 at a $44.1 \mathrm{kHz}$ sampling rate. The levels of the primary tones were $L_{1}=60 \mathrm{~dB}$ SPL, and $L_{2}=50 \mathrm{~dB}$ SPL. The $F_{2}$ frequency ranged from $1.5 \mathrm{kHz}$ to $10.2 \mathrm{kHz}$. The signals were sent to the headphone buffer (HB7) and next via an insert earphone (Etymotic Research, ER-2) were delivered to the ear canal. A low noise microphone (ER 10B + ) inserted into the ear canal in a soft plastic ear tip acquired the overall signal. For each subject the right ear was used as the test ear. During the measurements subjects were seated in a sound-treated room.

The contralateral signal was generated via an independent TDT channel at the level of $60 \mathrm{SPL}$ and was delivered to the contralateral ear. The levels of the primary tones and the contralateral signal were calibrated by low-noise probe microphones $(\mathrm{ER}-10 \mathrm{~B}+)$ placed in the external ear canals, before basic measurements.

Two modes of CS were used in the studies (without and with additional broadband noise to the contralateral ear). The contralateral signal was a wideband noise (bandwidth $0.2-10 \mathrm{kHz}$ ) modulated through two types of modulating signal (sine or rectangular wave form). The modulation depth was $100 \%$ for both sinusoidal and rectangular modulation. The modulation rate was 4 or $100 \mathrm{~Hz}$. The generation and acquisition of the DPOAE signal lasted $2 \mathrm{~s}$. It consisted of a $1 \mathrm{~s}$ acquisition in the absence of CS, and a $1 \mathrm{~s}$ recording in the presence of CS. The values of the DPOAE level changes were defined as the difference between the mean DPOAE levels with and without CS.

A bandpass filtering ( $8 \mathrm{~Hz}$ bandwidth, centred at frequency $\left.F_{\mathrm{DP}}\right)$ of the recorded signal was performed and the $F_{\mathrm{DP}}$ component level changes versus time were determined. The DPOAE levels with and without CS were determined as mean values in time intervals $(0.9 \mathrm{~s})$, which excluded a transient interval and comprised relatively steady level values of the DPOAE temporal record. The $F_{\mathrm{DP}}$ component was accepted as a significant response when the signal-to-noise ratio of the DPOAE exceeded $6 \mathrm{~dB}$.

\section{Results}

The OAE examinations were divided in two stages. In the first step DPOAE level changes were estimated for unmodulated CS. Figure 1 shows examples of DPgrams which demonstrate the emission level of the DPOAE as a function of $F_{2}$ frequency in the lower frequency range. 


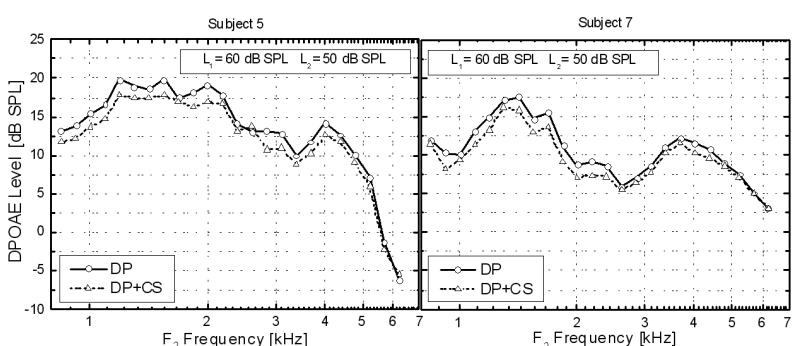

Fig. 1. Examples of DPgrams for two subjects. The solid line and triangles refer to DPOAE without CS.

The solid line and open circles refer to DPOAE level without CS, whereas the broken line and triangles depict the $F_{\mathrm{DP}}$ level with CS. Figure 1 indicates the lower $F_{\text {DP }}$ levels for CS, particularly for the $F_{2}$ frequency up to $3 \mathrm{kHz}$. This suppression effect ranged from a fraction of a decibel to few decibels, depending on the $F_{2}$ frequency. Sometimes a slight $F_{\mathrm{DP}}$ enhancement was also observed.

Changes in the DPOAE level for unmodulated CS were determined for three frequency ranges. These results are presented in Fig. 2, in the form of a scatterplot.

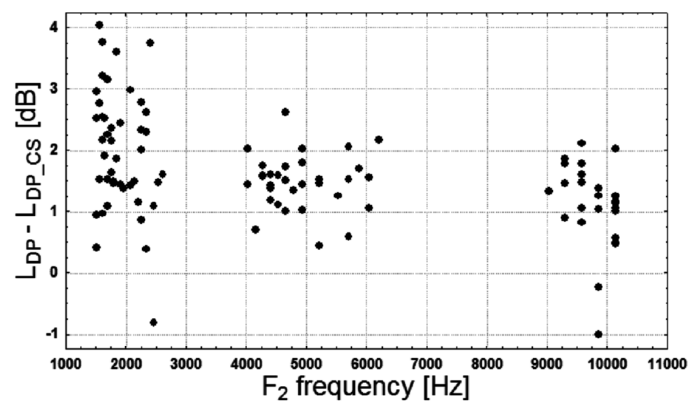

Fig. 2. Scatterplot of DPOAE level changes under the influence of CS.

It should be emphasized that positive DPOAE difference concerned suppression effect, while negative value indicated enhancement. In most cases positive DPOAE differences were observed, only in a few cases where enhancement effects were noticed. The greatest data scattering was found for the low frequency range. Additionally, in this frequency range the biggest suppression effect was observed (up to $4 \mathrm{~dB}$ ). The lower DPOAE suppression appeared in the middle and the highest frequency (around 5 and $10 \mathrm{kHz}$, respectively).

Generally it should be stated that DPOAE suppression is decreasing the function of the $F_{2}$ frequency for the whole $F_{2}$ frequency range. Additionally, in $96 \%$ cases the suppression effect was found. A one-way within-subjects analysis of variance (ANOVA) was performed. This analysis showed that the effect of the $F_{2}$ frequency $[F(37,63)=1.636, p=0.042]$ was statistically significant.

In the second stage of the research, amplitude modulated CS was applied. Again, a one-way within-subjects analysis of variance was performed. The factor was CS and contained the following factor codes: "am_4 Hz", "am_100 Hz", "rec_4 Hz" and "rec_100 Hz". These codes corresponded to the types of CS modulation used. The results of the study are presented in Fig. 3.

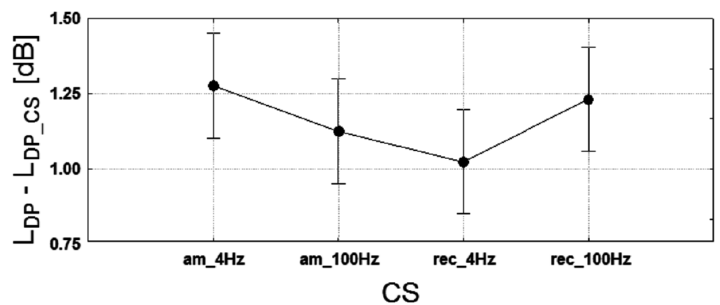

Fig. 3. Mean DPOAE suppression as a function of CS modulation type. Vertical bars indicate a $95 \%$ confidence interval.

The ANOVA analysis revealed that the type of modulation did not have an influence on suppression value $[F(3,366)=1.869, p=0.135]$. It is clear from Fig. 3 that the smallest suppression was obtained for the $4 \mathrm{~Hz}$ rectangular modulation and the largest for the $4 \mathrm{~Hz}$ sinusoidal modulation. However, these differences were not statistically significant.

Comparison of the research findings from the second and third stage enables an overall view of the influence of amplitude modulation on the change of DPOAE amplitude during CS. In this case, the ANOVA analysis showed very statistically significant differences between the CS types (unmodulated or amplitude modulated) $[F(4,466)=9.629, p=0.000]$. This is shown in Fig. 4 .

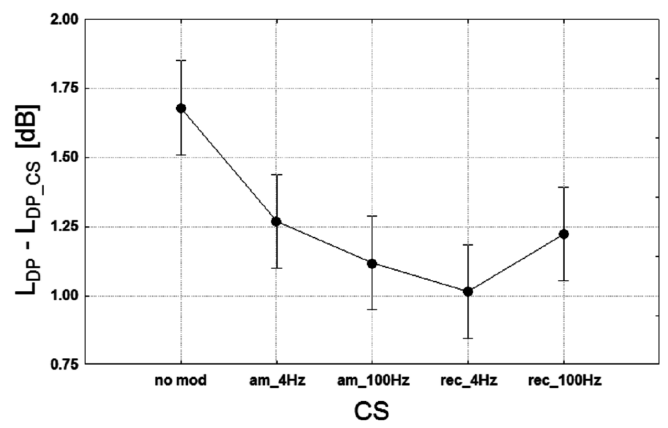

Fig. 4. Mean DPOAE suppression as a function of CS types (unmodulated or amplitude modulated). Vertical bars indicate a $95 \%$ confidence interval.

The DPOAE level reduction reached the highest value of about $1.7 \mathrm{~dB}$ for the unmodulated CS.

\section{Discussion}

The available research findings in the literature indicate the significant influence of contralateral modulation of the contralateral signal on changes in the OAE level. Some authors claim that this influence depends both on 
the modulation rate as well as the modulation depth [19, 21]. Maison et al. [21] used sinusoidally modulated broadband noise, presented contralaterally with modulation rates from 50 to $800 \mathrm{~Hz}$. They stated that the largest TEOAE suppression effect was observed at the $100 \mathrm{~Hz}$ modulation rate and this suppression was larger than for unmodulated CS. The results of this work did not confirm conclusions from the examinations conducted by Maison et al. [21]. Independently of the modulation type and modulation rate, the suppression effect was lower than for the unmodulated CS. Considering the conclusions from Harrison et al. paper [22], for fast changes of the CS amplitude envelope, the suppression should be similar to that of the unmodulated signal. The results showed in this paper did not confirm this hypothesis. Applying broadband noise as a carrier signal causes the long-term spectrum of the signal to be almost the same as the unmodulated signal. In other words, changes of suppression can result only from temporal fluctuations in the contralateral signal.

The results presented in this paper allow the following conclusions to be formulated:

- For unmodulated contralateral broadband noise, the DPOAE suppression is decreasing the function of the $f_{2}$ frequency for the whole frequency range.

- The modulation rate and the type of the modulation did not have an influence on the suppression value $[F(3,366)=1.869, p=0.135]$ during $\mathrm{CS}$.

- The DPOAE level reduction reached a higher value for the unmodulated contralateral signal than for the modulated. This difference is statistically significant $[F(4,466)=9.629, p=0.000]$.

Examinations concerning temporal analysis of the envelope of the $F_{\mathrm{DP}}$ component in CS conditions are being continued. Also a spectral analysis showed that in the envelope of the non-linear component, the modulation rate of the contralateral signal was reproduced.

\section{Acknowledgments}

This work was supported by a grant from the Ministry of Science and Higher Education No. N N518 405438.

\section{References}

[1] D.T. Kemp, Hear Res. 22, 95 (1986).

[2] R. Probst, B.L. Lonsbury-Martin, G.K. Martin, J. Acoust. Soc. Am. 89, 2027 (1991).

[3] X.M. Sun, J. Acoust. Soc. Am. 123, 4310 (2008).

[4] S. Chery-Croze, A. Moulin, L. Collet, Hear Res. 68, 53 (1993).

[5] G. Lisowska, J. Smurzynski, K. Morawski, G. Namyslowski, R. Probst, Acta Otolaryngol. 122, 613 (2002).

[6] J.J. Guinan, Jr., Ear Hear 27, 589 (2006).

[7] C. Abdala, Hear Res. 121, 125 (1998).

[8] M.P. Gorga, S.T. Neely, P.A. Dorn, D. Konrad-Martin, J. Acoust. Soc. Am. 111, 271 (2002).

[9] F. Zhang, F.A. Boettcher, X.M. Sun, Int. J. Audiol. 46, 187 (2007).

[10] A.L. James, R.J. Mount, R.V. Harrison, Clin. Otolaryngol. Appl. Sci. 27, 106 (2002).

[11] D.W. Purcell, B.E. Butler, T.J. Saunders, P. Allen, J. Acoust. Soc. Am. 124, 2133 (2008).

[12] E. Ozimek, A. Wicher, in: XXVIII Int. Congress of Audiology, Innsbruck (Austria), 2006, p. 43.

[13] A. Wicher, Ear Hear 2011, submitted for publication.

[14] J. Muller, T. Janssen, G. Heppelmann, W. Wagner, J. Acoust. Soc. Am. 118, 3747 (2005).

[15] D.S. Velenovsky, T.J. Glattke, Hear Res. 164, 39 (2002).

[16] S.R. Atcherson, M.J. Martin, R. Lintvedt, Neurosci. Lett. 438, 107 (2008).

[17] J.J. Guinan, Jr., B.C. Backus, W. Lilaonitkul, V. Aharonson, J. Assoc. Res. Otolaryngol. 4, 521 (2003).

[18] J.J. Guinan, in: The Cochlea, Eds. P.J. Dallos, A.N. Popper, R.R. Fay, Springer-Verlag, New York 1996, p. 435

[19] S. Maison, C. Micheyl, L. Collet, J. Neurophysiol. 77, 1759 (1997).

[20] C. Micheyl, S. Maison, R.P. Carlyon, G. Andeol, L. Collet, J. Acoust. Soc. Am. 105, 293 (1999).

[21] S. Maison, C. Micheyl, L. Collet, Neuroscience 91, 133 (1999).

[22] R.V. Harrison, A. Sharma, T. Brown, S. Jiwani, A.L. James, Acta Otolaryngol. 128, 404 (2008). 